\section{Congenital absence of the left circumflex artery in a patient presenting with acute inferior myocardial infarction of}

The absence of the left circumflex artery (LCX) with the superdominant right coronary artery (RCA) is an uncommon congenital coronary artery anomaly. Further, the coexistence of the absence of the LCX and acute inferior myocardial infarction is extremely rare.

A 50-year-old female patient with a history of hypertension was admitted to the emergency department with a complaint of ongoing precordial chest pain that had started 45 minutes earlier. Physical examination was normal except for bradycardia with a heart rate of 48 . Electrocardiography showed complete atrioventricular block with ST-segment elevation in the inferior leads, with reciprocal ST-segment depression in the anterolateral leads (Fig. 1a). Conventional coronary angiography revealed a total occlusion of the mid segment of the RCA (Fig. 1b, Video 1), noncritical lesion of the left anterior descending artery (Fig. 1c, Video 2), and an absent LCX that could also not be shown with the aortography. Immediate primary percutaneous intervention with stenting to the RCA was done and distal flow was achieved, which showed a superdominant RCA that supplies the LCX territory (Fig. 1d, Video 3). Multidetector coronary computed tomography was performed three days after hospitalization, and it confirmed the absence of the LCX (Fig. 2a, $2 \mathrm{~b}$ ) and superdominant RCA, which goes through the left atrioventricular groove and supplies the posterolateral and inferior portions of the left ventricle (Fig. 2c, 2d). Following the uneventful procedure, the chest pain disappeared, the bradycardia resolved, and a sinus rhythm was achieved.

It is important to differentiate the total orifice occlusion of the LCX from the absence of it, and physicians should be familiar with these anatomic variants to avoid any iatrogenic injuries to these anomalous vessels and to decide on proper revascularization strategies, particularly in emergency conditions.

Informed consent: Written informed consent was obtained from the patient for the publication.

Video 1. Right coronary angiogram shows the total occlusion of the mid segment of the right coronary artery.

Video 2. Left coronary angiogram reveals the absence of the left circumflex artery.

Video 3. Right coronary angiogram shows superdominant right coronary artery with large posterolateral branches supplying the area of the left circumflex territory.
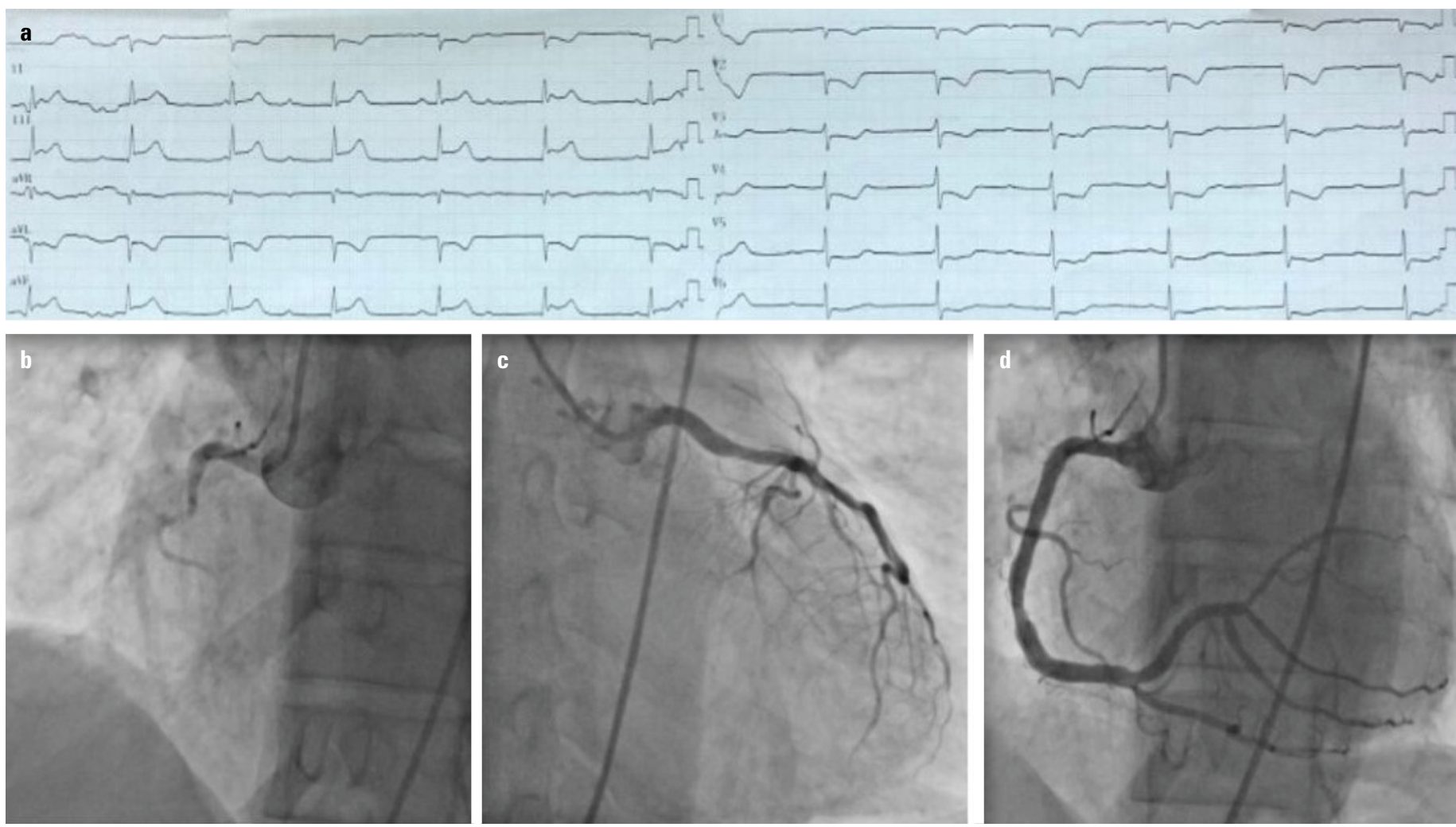

Figure 1. ECG shows acute inferior myocardial infarction (a). Right coronary angiogram (CA) showing the total occlusion of the mid segment of the right coronary artery (RCA) (b) and superdominant RCA (d). Left CA demonstrates the absence of the left circumflex artery (c) 

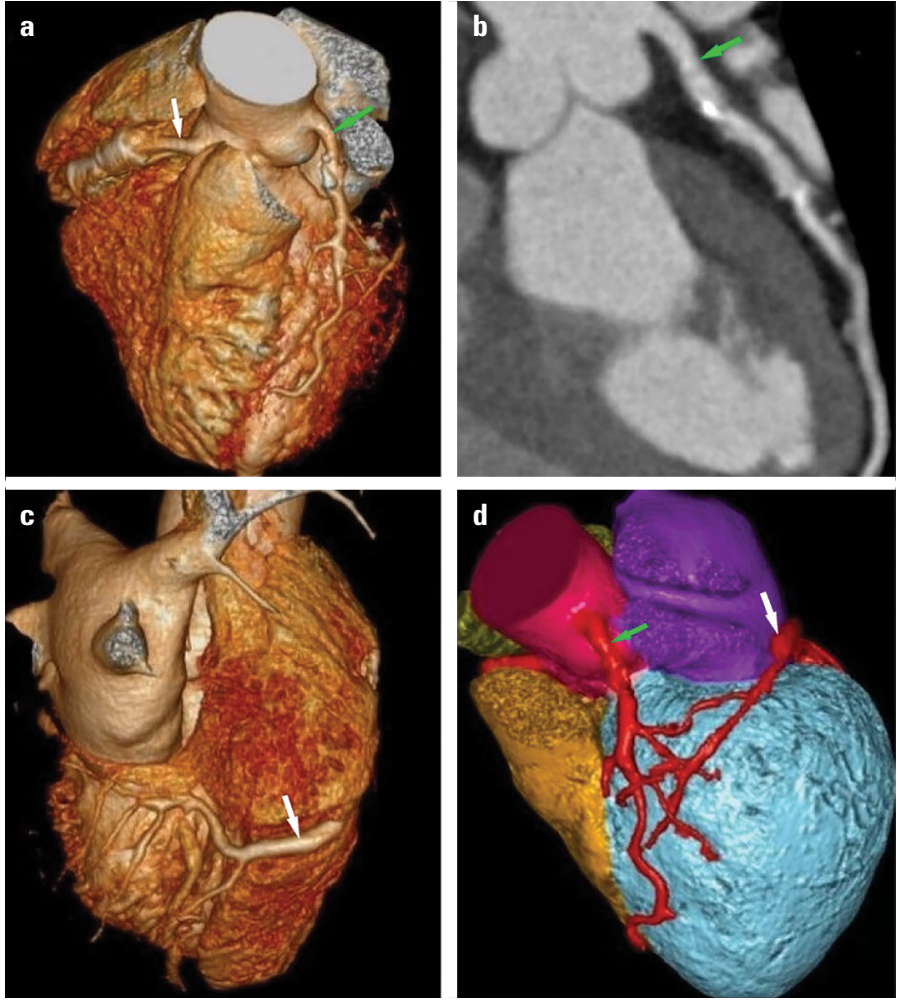

Figure 2. Multidetector coronary computed tomography reveals long left main coronary artery, the absence of the left circumflex artery (green arrows) (a, b), and superdominant right coronary artery (white arrows) with large posterolateral branches (c, d)

(D) Orhan İnce, (D) Kamil Gülşen', (D) Sevil Tuğrul, (D) İrfan Şahin, (iD) Ertuğrul Okuyan

Department of Cardiology, Health and Science University, Bağcılar Training and Research Hospital; İstanbul-Turkey

'Department of Cardiology, Health and Science University, Kartal Koşuyolu Training and Research Hospital; İstanbul-Turkey

Address for Correspondence: Dr. Orhan Ince,

Sağlık Bilimleri Üniversitesi,

Bağcılar Eğitim ve Araştırma Hastanesi,

Kardiyoloji Anabilim Dalı,

İstanbul-Türkiye

Phone: +90 5302287438 - 2124404000

E-mail: drorhanince@gmail.com

(C) Copyright 2020 by Turkish Society of Cardiology - Available online

at www.anatoljcardiol.com

DOI:10.14744/AnatolJCardiol.2020.62605

\section{A rocking motion of a patent foramen ovale device}

A 58-year-old man, who experienced recurrent strokes while on aspirin therapy, was admitted for further evaluation. The patient's electrocardiogram showed normal sinus rhythm, and transthoracic echocardiography revealed normal cardiac functions with mild tricuspid regurgitation and a floppy interatrial sep- tum. Transesophageal echocardiography (TEE) revealed a patent foramen ovale (PFO) with an atrial septal aneurysm (Fig. 1, Video 1). Therefore, a PFO closure was planned.

With the patient under general anesthesia, a 25-mm Amplatzer PFO Occluder (St. Jude Medical, Plymouth, MN, USA) was placed at the correct position and subsequently released after the final assessment of both TEE and fluoroscopy. However, after deployment, fluoroscopy revealed that the PFO Occluder had a rocking motion (Video 2). The motion was synchronized with breathing, possibly attributing it to the cardiac chamber expansion and increased cardiac preload during inspiration as the underlying mechanisms. It is hypothesized that the rocking motion could be exaggerated by increased preload, owing to colloid infusion before venous puncture. The patient was then conservatively followed. On the second day, control fluoroscopy showed that the device was in a steady position (Video 3).
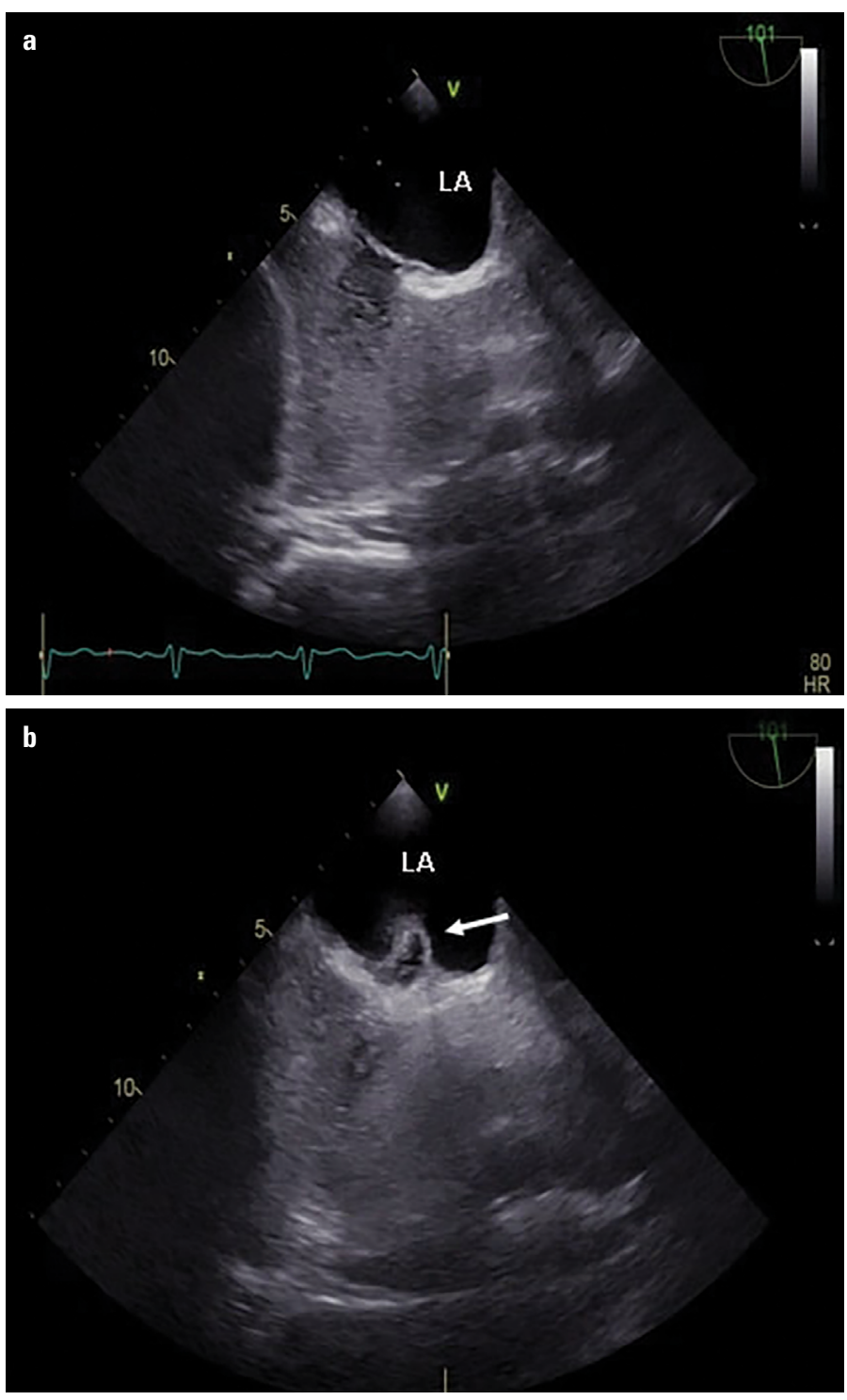

Figure 1. (a) TEE image of the PFO device. (b) TEE image of the large atrial septal aneurysm. The arrow indicates that the atrial septal aneurysm has large amplitude movements bulging to the left atrium 